\title{
Pooled analysis of two case-control studies on the use of cellular and cordless telephones and the risk of benign brain tumours diagnosed during 1997-2003
}

\author{
LENNART HARDELL ${ }^{1,2}$, MICHAEL CARLBERG ${ }^{1}$ and KJELL HANSSON MILD ${ }^{2,3}$ \\ ${ }^{1}$ Department of Oncology, University Hospital, SE-701 85 Örebro; ${ }^{2}$ Department of Natural Sciences, \\ Örebro University, SE-701 82 Örebro; ${ }^{3}$ National Institute for Working Life, SE-907 13 Umeå, Sweden
}

Received September 22, 2005; Accepted November 8, 2005

\begin{abstract}
The use of cellular and cordless telephones and the risk of brain tumours is of concern since the brain is a high exposure area. We present the results of a pooled analysis of two case-control studies on benign brain tumours diagnosed during 1997-2003 including answers from 1,254 (88\%) cases and 2,162 (89\%) controls aged 20-80 years. For acoustic neuroma, the use of analogue cellular phones gave an odds ratio (OR) of 2.9 and a $95 \%$ confidence interval (CI) of 2.04.3; for digital cellular phones, $\mathrm{OR}=1.5 ; 95 \% \mathrm{CI}=1.1-2.1$; and for cordless telephones, $\mathrm{OR}=1.5,95 \% \mathrm{CI}=1.04-2.0$. The highest OR was found for analogue phones with a latency period of $>15$ years; $\mathrm{OR}=3.8,95 \% \mathrm{CI}=1.4-10$. Regarding meningioma, the results were as follows: for analogue phones, $\mathrm{OR}=1.3,95 \% \mathrm{CI}=0.99-1.7$; for digital phones, $\mathrm{OR}=1.1,95 \%$ $\mathrm{CI}=0.9-1.3$; and for cordless phones, $\mathrm{OR}=1.1,95 \% \mathrm{CI}=0.9$ 1.4. In the multivariate analysis, a significantly increased risk of acoustic neuroma was found with the use of analogue phones.
\end{abstract}

\section{Introduction}

The use of cellular and cordless telephones is widespread and increasing in society. A potential association between cellular and cordless telephones and health effects is of concern and has been discussed in several articles during recent years (1-3). Of special concern is the risk of brain tumours since this is a part of the body with high exposure during phone calls compared with other parts.

Cellular telephones emit radio frequency signals during calls. Exposure is characterized through the specific absorption rate (SAR) expressed as watt/kg. Analogue (NMT; Nordic Mobile Telephone System) phones operating at 450 MegaHertz

Correspondence to: Professor Lennart Hardell, Department of Oncology, University Hospital, SE-701 85 Örebro, Sweden

E-mail: lennart.hardell@orebroll.se

Key words: acoustic neuroma, analogue cellular telephones
(MHz) were introduced in Sweden in 1981. At first, they were usually used in cars with a fixed external antenna. Portable NMT 450 phones were introduced in 1984. Analogue phones using $900 \mathrm{MHz}$ (NMT 900) were used in Sweden between 1986 and 2000. The digital system (GSM; Global System for Mobile Communication) started in 1991 and has during recent years dramatically increased to become the most common phone type. This system uses dual band, 900 and 1,800 MHz, for communication. From 2003, the third generation of mobile phones, $3 \mathrm{G}$ or UMTS (Universal Mobile Telecommunication System), was introduced in Sweden, operating at $1,900 \mathrm{MHz}$.

Desktop cordless phones using the analogue system in the 800-900 MHz RF have been used in Sweden since 1988. Digital cordless telephones (DECT) that operate at 1,900 MHz have been used since 1991 .

Acoustic neuroma might be a 'signal' tumor for association with cellular and cordless telephones, since it is located in an area with high exposure during calls. The tumour risk would be higher on the same side of the head as the exposure to the RF-field (ipsilateral exposure).

In 1999, we published our first study on this topic with cases and controls from between 1994 and 1996 (4). The analyses were based on answers from 209 (90\%) of the cases and 425 (91\%) of the controls. Overall, we did not find an increased risk. However, for ipsilateral exposure, we saw a somewhat higher risk, although based on few exposed subjects $(4,5)$. No conclusions could be drawn from that study due to the low numbers and short latency periods.

Our next case-control study was larger and the responding numbers were for 1,429 cases $(88 \%)$ and 1,470 controls (91\%) recruited during January 1, 1997, and June 30, 2000. We modified the questionnaire somewhat between the two studies to assess exposure as carefully as possible. For all brain tumours, we found an increased risk for analogue phones that was most pronounced in the group with a $>10$-year latency period $(6,7)$. Moreover, the risk was highest for analogue and digital cellular telephones with ipsilateral exposure, especially for high-grade astrocytoma. We found no association for meningioma. Regarding acoustic neuroma, a high risk was calculated for the use of analogue phones (6-8).

In our third study, we used the same questionnaire and study methods as in the second in order to be able to pool these 
two studies for a larger amount of study material with a longer time of use for both cellular and cordless phones $(9,10)$. This study was conducted from July 1, 2000, until December 31, 2003. The study area consisted of Uppsala/Örebro and Linköping medical regions in Sweden. This time, Stockholm and Gothenburg medical regions were not included since the WHO Interphone study on the same issue was performed during part of this time in these regions. Thus, there was no overlap of cases between any of our three studies on this topic or the Swedish part of the Interphone study $(11,12)$.

Here, we present results for benign brain tumours in a pooled analysis of our second and third studies on this topic. All controls from the second and third studies are used as a reference entity.

\section{Materials and methods}

In previous studies, we have presented details on the study methods so only a short presentation is given here. Ethics committees approved the studies. Both men and women, aged 20-80 years at the time of diagnosis as defined according to the date of the histopathology report, were included. In total, 3,729 cases were reported in a consecutive manner from the regional cancer registries diagnosed during the inclusion period. Subjects that did not meet the study prerequisites were excluded, i.e. brain metastases or wrong reporting to the registry $(n=288)$, wrong year for diagnosis $(n=73)$, missing histopathology $(n=5)$, not resident in the study area $(n=14)$, deceased $(n=745)$, physician refusal $(n=81)$, not able to participate $(n=84)$ and unknown address $(n=2)$, in a total of 1,292 cases. The final pooled study included 2,437 cases or $65 \%$ of those initially reported. Of these, 1,429 had a benign brain tumor.

We drew one control subject matched on age and sex and residing in the study area to each case from the Swedish population registry, which covers the whole population with unique id-numbers and current addresses. Any change in residence can be traced in the registry. Thus, 2,437 controls were recruited.

Assessment of exposure. Different environmental and occupational exposures were assessed by using a 20-page questionnaire sent to the study subjects. It contained questions on complete working history, exposure to different agents, smoking habits, etc. Regarding the use of cellular telephones, we asked for the first year of use, type of phone (analogue with prefix 010 , digital with prefix 07 ), mean minutes of daily use over the years, use in a car with external antenna or a hands-free (both calculated as unexposed) and ear most frequently used. Similar questions dealt with the use of cordless telephones.

At most, two reminders were sent if the questionnaire was not returned. Trained interviewers using a structured protocol made supplementary phone interviews, if necessary. The ear that had been most frequently used over the years was asked for. A change in the used ear might have occurred, e.g. in the case of acoustic neuroma, due to hearing loss. The interviewer checked this information but we also sent an additional letter and asked all study subjects using cellular or cordless telephones to clarify this issue in detail.
An id-code that did not reveal whether the individual was a case or a control was given to each questionnaire. Thus, interviews and coding of data for the statistical analysis were blinded to case or control status. Both clinical and pathology reports were sent to the cancer registry in Sweden. We obtained such data from cancer registries and histopathological departments in the study area. All cases were given a diagnosis based on histopathological examination. Tumor localization was obtained by data in the cancer registries or if missing or unclear from neuroradiology investigations. We obtained copies of records after informed consent from the cases.

Statistical methods. Odds ratios (OR) and 95\% confidence intervals (CI) (Stata/SE 8.2 for Windows; StataCorp, College Station, TX) were calculated using unconditional logistic regression analysis. The unexposed category consisted of subjects that had not used cellular or cordless phones. The exposed cases and controls were divided according to phone type, analogue, digital and cordless. We calculated the OR and $95 \% \mathrm{CI}$ for the use of only one of these phone types and for different combinations. Adjustment was made for sex, age, socio-economic index (SEI)-code and year of diagnosis. Thereby, the same year as for the case was used for the corresponding control. Adjustment for year of diagnosis was made in order to avoid bias in exposure since all controls, both to malignant and benign brain tumor cases, were used in the analysis. We used age as a continuous variable in the analysis. Latency or tumor induction period was analysed using three time periods, $>1-5,>5-10$ and $>10$ years from the first use of a cellular or cordless telephone until diagnosis. In the dose-response calculations, the median number of cumulative lifetime use in hours among controls was used as a cut-off. Note that overall results for all latency groups were calculated in one analysis whereas dose-response was analysed separately for each latency category.

\section{Results}

In total, 1,254 (88\%) cases and 2,162 (89\%) controls participated. Of these, 916 (73\%) had meningioma, 243 (19\%) had acoustic neuroma and $96(8 \%)$ had other types of benign brain tumours. One case had both meningioma and acoustic neuroma and was included in calculations of OR and $95 \% \mathrm{CI}$ for both diseases. We display the results for cumulative use in hours for the different phone types in Table I. Regarding acoustic neuroma, the highest OR was calculated for cellular and cordless telephones for subjects with $>1,000 \mathrm{~h}$ of cumulative use of the respective phone. The same pattern was found for meningioma and the use of cordless phones.

In Table II, we give the results for the different phone types according to latency period and cumulative number of hours divided into two groups based on the median number of hours among the controls. For meningioma, a somewhat increased OR was found with a longer latency period, the highest with a $>10$-year latency period. For acoustic neuroma, the use of analogue phones produced $\mathrm{OR}=2.9,95 \% \mathrm{CI}=2.0-4.3$; digital phones, $\mathrm{OR}=1.5,95 \% \mathrm{CI}=1.1-2.1$; and cordless telephones, $\mathrm{OR}=1.5,95 \% \mathrm{CI}=1.04-2.0$. The $\mathrm{OR}$ was higher in the group with a $>5$ - to 10 -year latency compared with a shorter time. Only one case had used a digital phone for $>10$ years 
Table I. Odds ratio (OR) and 95\% confidence interval (CI) for cumulative lifetime use in hours of analogue and digital cellular telephones, cordless telephones and any combination of the three phone types. ${ }^{\mathrm{a}}$

\begin{tabular}{|c|c|c|c|c|c|c|c|c|c|}
\hline & \multicolumn{3}{|c|}{$1-500 \mathrm{~h}$} & \multicolumn{3}{|c|}{$501-1000 \mathrm{~h}$} & \multicolumn{3}{|c|}{$>1000 \mathrm{~h}$} \\
\hline & $\mathrm{Ca} / \mathrm{Co}$ & OR & $95 \% \mathrm{CI}$ & $\mathrm{Ca} / \mathrm{Co}$ & OR & $95 \% \mathrm{CI}$ & $\mathrm{Ca} / \mathrm{Co}$ & OR & $95 \% \mathrm{CI}$ \\
\hline \multicolumn{10}{|l|}{ Benign } \\
\hline Analogue & $169 / 252$ & 1.6 & $1.2-2.0$ & $17 / 29$ & 1.7 & $0.9-3.1$ & $13 / 16$ & 2.2 & $1.02-4.7$ \\
\hline Digital & $384 / 667$ & 1.2 & $0.96-1.4$ & $30 / 64$ & 1.1 & $0.7-1.8$ & $23 / 45$ & 1.2 & $0.7-2.1$ \\
\hline Cordless & $269 / 502$ & 1.1 & $0.9-1.3$ & $70 / 97$ & 1.5 & $1.1-2.1$ & $84 / 102$ & 1.6 & $1.2-2.2$ \\
\hline Total, any combination & $450 / 831$ & 1.1 & $0.9-1.3$ & $98 / 152$ & 1.4 & $1.03-1.8$ & $129 / 189$ & 1.5 & $1.1-1.9$ \\
\hline \multicolumn{10}{|l|}{ Meningioma } \\
\hline Analogue & $99 / 252$ & 1.3 & $0.98-1.7$ & $8 / 29$ & 1.1 & $0.5-2.6$ & $6 / 16$ & 1.4 & $0.5-3.8$ \\
\hline Digital & $268 / 667$ & 1.1 & $0.9-1.3$ & $18 / 64$ & 1.0 & $0.6-1.8$ & $9 / 45$ & 0.7 & $0.3-1.4$ \\
\hline Cordless & $185 / 502$ & 1.0 & $0.8-1.3$ & $49 / 97$ & 1.5 & $1.003-2.2$ & $60 / 102$ & 1.6 & $1.1-2.2$ \\
\hline Total, any combination & $310 / 831$ & 1.0 & $0.8-1.2$ & $66 / 152$ & 1.3 & 0.9-1.8 & $85 / 189$ & 1.3 & $0.99-1.8$ \\
\hline \multicolumn{10}{|l|}{ Acoustic neuroma } \\
\hline Analogue & $55 / 252$ & 2.8 & $1.8-4.2$ & $7 / 29$ & 3.3 & $1.3-8.0$ & $6 / 16$ & 5.1 & $1.9-14$ \\
\hline Digital & $83 / 667$ & 1.4 & $0.99-2.0$ & $10 / 64$ & 1.8 & $0.8-3.8$ & $12 / 45$ & 3.1 & $1.5-6.4$ \\
\hline Cordless & $60 / 502$ & 1.3 & $0.9-1.9$ & $15 / 97$ & 1.6 & $0.9-3.0$ & $21 / 102$ & 2.1 & $1.2-3.7$ \\
\hline Total, any combination & $97 / 831$ & 1.3 & $0.96-1.8$ & $22 / 152$ & 1.6 & $0.96-2.8$ & $36 / 189$ & 2.2 & $1.4-3.4$ \\
\hline \multicolumn{10}{|l|}{ Other benign } \\
\hline Analogue & $16 / 252$ & 1.5 & $0.8-3.0$ & $2 / 29$ & 2.0 & $0.4-9.5$ & $1 / 16$ & 2.1 & $0.3-17$ \\
\hline Digital & $34 / 667$ & 1.5 & $0.9-2.7$ & $2 / 64$ & 0.6 & $0.1-2.7$ & $2 / 45$ & 1.1 & $0.2-4.8$ \\
\hline Cordless & $24 / 502$ & 1.4 & $0.8-2.6$ & 6/97 & 1.7 & $0.7-4.4$ & $4 / 102$ & 1.2 & $0.4-3.6$ \\
\hline Total, any combination & $43 / 831$ & 1.5 & $0.9-2.4$ & $10 / 152$ & 1.7 & $0.8-3.7$ & $9 / 189$ & 1.3 & $0.6-2.8$ \\
\hline
\end{tabular}

${ }^{a}$ Number of exposed cases $(\mathrm{Ca})$ and controls $(\mathrm{Co})$ are given. Unconditional logistic regression analysis adjusted for age, sex, socio-economic index and year of diagnosis was used. Trend, benign: analogue, $\mathrm{p}=0.71$; digital, $\mathrm{p}=0.97$; cordless, $\mathrm{p}=0.02 ;$ total, $\mathrm{p}=0.02$. Trend, meningioma: analogue, $\mathrm{p}=0.93$; digital, $\mathrm{p}=0.50$; cordless, $\mathrm{p}=0.02$; total, $\mathrm{p}=0.04$. Trend, acoustic neuroma: analogue, $\mathrm{p}=0.48$; digital, $\mathrm{p}=0.08$; cordless, $\mathrm{p}=0.17$; total, $\mathrm{p}=0.07$. Trend, other benign: analogue, $\mathrm{p}=0.91$; digital, $\mathrm{p}=0.41$; cordless, $\mathrm{p}=0.86$; total, $\mathrm{p}=0.84$.

and 4 cases had used a cordless telephone for $>10$ years. Regarding analogue phones, a latency period of $>10-15$ years yielded $\mathrm{OR}=2.9,95 \% \mathrm{CI}=1.4-6.0$ ( $\mathrm{n}=13$ cases, 62 controls) and $>15$ years yielded $\mathrm{OR}=3.8,95 \% \mathrm{CI}=1.4-10$ ( $\mathrm{n}=6$ cases, 22 controls). Digital cellular phones gave a significantly increased risk in the group of other benign tumours with a 10-year latency period but based on only 4 cases.

Regarding meningioma, the OR was somewhat higher for ipsilateral use of cellular or cordless phones than for contralateral, and was of borderline significance for digital phones (Table III). Also, for acoustic neuroma, the most pronounced effect on OR was seen for ipsilateral use. Thus, for analogue phones, ipsilateral use yielded $\mathrm{OR}=3.0,95 \% \mathrm{CI}=1.9-5.0$, but contralateral and varying ipsi-/contralateral use also increased the risk. For other types of benign brain tumours, these calculations were based on rather low numbers of cases to permit clear conclusions.

In the multivariate analysis, as displayed in Table IV, no significantly increased risk was found for meningioma for the studied phone. For acoustic neuroma, we found an OR of 2.5 and $95 \%$ CI of 1.8-3.5 for the use of analogue phones. The result was similar in the latency groups of $>5-10$ years and $>10$ years. The use of digital cellular telephones or cordless telephones yielded no significantly increased risk. In the group with other types of benign brain tumours, digital cellular phones gave a significantly increased risk with a $>10$-year latency period but based on few exposed cases.

Table V shows our analysis of OR for the use of only one type of phone and for different combinations. For meningioma, the OR was highest for subjects that reported the use of several types of phones. A similar pattern was found for acoustic neuroma with a significantly increased OR for any combination of cellular and cordless telephones.

We analysed the association between use of cellular and cordless telephones for different age groups based on first use of the respective phone (Table VI). The OR was highest for subjects in the $<20$ years age group for use of both analogue or digital cellular telephones, although based on low numbers. 
Table II. Number of exposed cases (Ca) with benign brain tumours and controls (Co), odds ratio (OR) and 95\% confidence interval (CI) for use of cellular or cordless telephones. ${ }^{a}$

\begin{tabular}{|c|c|c|c|c|c|c|c|c|}
\hline & \multicolumn{2}{|c|}{$>1$ - to 5-year latency } & \multicolumn{2}{|c|}{$>5$ - to 10 -year latency } & \multicolumn{2}{|c|}{$>10$-year latency } & \multicolumn{2}{|c|}{ Total, >1-year latency } \\
\hline & $\mathrm{Ca} / \mathrm{Co}$ & $\mathrm{OR}, \mathrm{CI}$ & $\mathrm{Ca} / \mathrm{Co}$ & $\mathrm{OR}, \mathrm{CI}$ & $\mathrm{Ca} / \mathrm{Co}$ & $\mathrm{OR}, \mathrm{CI}$ & $\mathrm{Ca} / \mathrm{Co}$ & $\mathrm{OR}, \mathrm{CI}$ \\
\hline \multicolumn{9}{|c|}{$\begin{array}{l}\text { Benign }(n=1254, \\
577 \text { unexposed })\end{array}$} \\
\hline Analogue & $52 / 86$ & $\begin{array}{l}1.4 \\
0.9-2.0\end{array}$ & $90 / 127$ & $\begin{array}{l}1.7 \\
1.2-2.3\end{array}$ & $57 / 84$ & $\begin{array}{l}1.8 \\
1.2-2.6\end{array}$ & $199 / 297$ & $\begin{array}{l}1.6 \\
1.3-2.0\end{array}$ \\
\hline$\leq 85 \mathrm{~h}$ & $40 / 67$ & $\begin{array}{l}1.3 \\
0.8-2.0\end{array}$ & $46 / 63$ & $\begin{array}{l}1.6 \\
1.1-2.4\end{array}$ & $20 / 26$ & $\begin{array}{l}2.0 \\
1.1-3.7\end{array}$ & $106 / 156$ & $\begin{array}{l}1.5 \\
1.1-2.0\end{array}$ \\
\hline$>85 \mathrm{~h}$ & $12 / 19$ & $\begin{array}{l}1.6 \\
0.7-3.4\end{array}$ & $44 / 64$ & $\begin{array}{l}1.7 \\
1.1-2.7\end{array}$ & $37 / 58$ & $\begin{array}{l}1.8 \\
1.1-2.8\end{array}$ & $93 / 141$ & $\begin{array}{l}1.7 \\
1.3-2.4\end{array}$ \\
\hline Digital & $323 / 581$ & $\begin{array}{l}1.1 \\
0.9-1.4\end{array}$ & $101 / 177$ & $\begin{array}{l}1.2 \\
0.9-1.7\end{array}$ & $13 / 18$ & $\begin{array}{l}1.6 \\
0.8-3.5\end{array}$ & $437 / 776$ & $\begin{array}{l}1.2 \\
0.96-1.4\end{array}$ \\
\hline$\leq 64 \mathrm{~h}$ & $208 / 349$ & $\begin{array}{l}1.2 \\
0.9-1.5\end{array}$ & $37 / 70$ & $\begin{array}{l}1.1 \\
0.7-1.7\end{array}$ & $0 / 0$ & - & $245 / 419$ & $\begin{array}{l}1.1 \\
0.9-1.4\end{array}$ \\
\hline$>64 \mathrm{~h}$ & $115 / 232$ & $\begin{array}{l}1.1 \\
0.9-1.5\end{array}$ & $64 / 107$ & $\begin{array}{l}1.5 \\
1.04-2.2\end{array}$ & $13 / 18$ & $\begin{array}{l}1.6 \\
0.8-3.5\end{array}$ & $192 / 357$ & $\begin{array}{l}1.2 \\
0.96-1.5\end{array}$ \\
\hline Cordless & $250 / 437$ & $\begin{array}{l}1.1 \\
0.9-1.4\end{array}$ & $145 / 219$ & $\begin{array}{l}1.4 \\
1.1-1.7\end{array}$ & $28 / 45$ & $\begin{array}{l}1.4 \\
0.8-2.3\end{array}$ & $423 / 701$ & $\begin{array}{l}1.2 \\
1.01-1.4\end{array}$ \\
\hline$\leq 195 \mathrm{~h}$ & $137 / 260$ & $\begin{array}{l}1.0 \\
0.8-1.3\end{array}$ & $43 / 74$ & $\begin{array}{l}1.3 \\
0.8-1.9\end{array}$ & $3 / 17$ & $\begin{array}{l}0.6 \\
0.2-2.1\end{array}$ & $183 / 351$ & $\begin{array}{l}1.0 \\
0.8-1.3\end{array}$ \\
\hline$>195 \mathrm{~h}$ & $113 / 177$ & $\begin{array}{l}1.2 \\
0.9-1.6\end{array}$ & $102 / 145$ & $\begin{array}{l}1.6 \\
1.2-2.1\end{array}$ & $25 / 28$ & $\begin{array}{l}2.1 \\
1.1-3.7\end{array}$ & $240 / 350$ & $\begin{array}{l}1.4 \\
1.1-1.7\end{array}$ \\
\hline \multicolumn{9}{|c|}{$\begin{array}{l}\text { Meningioma }(n=916 \text {, } \\
455 \text { unexposed })\end{array}$} \\
\hline Analogue & $32 / 86$ & $\begin{array}{l}1.2 \\
0.8-1.8\end{array}$ & $47 / 127$ & $\begin{array}{l}1.2 \\
0.8-1.8\end{array}$ & $34 / 84$ & $\begin{array}{l}1.6 \\
1.02-2.5\end{array}$ & $113 / 297$ & $\begin{array}{l}1.3 \\
0.99-1.7\end{array}$ \\
\hline$\leq 85 \mathrm{~h}$ & $25 / 67$ & $\begin{array}{l}1.1 \\
0.7-1.8\end{array}$ & $28 / 63$ & $\begin{array}{l}1.3 \\
0.8-2.2\end{array}$ & $12 / 26$ & $\begin{array}{l}1.8 \\
0.9-3.7\end{array}$ & $65 / 156$ & $\begin{array}{l}1.3 \\
0.9-1.8\end{array}$ \\
\hline$>85 \mathrm{~h}$ & $7 / 19$ & $\begin{array}{l}1.4 \\
0.6-3.5\end{array}$ & $19 / 64$ & $\begin{array}{l}1.1 \\
0.6-1.9\end{array}$ & $22 / 58$ & $\begin{array}{l}1.6 \\
0.9-2.7\end{array}$ & $48 / 141$ & $\begin{array}{l}1.3 \\
0.9-1.9\end{array}$ \\
\hline Digital & $220 / 581$ & $\begin{array}{l}1.0 \\
0.8-1.3\end{array}$ & $67 / 177$ & $\begin{array}{l}1.1 \\
0.8-1.6\end{array}$ & $8 / 18$ & $\begin{array}{l}1.3 \\
0.5-3.2\end{array}$ & $295 / 776$ & $\begin{array}{l}1.1 \\
0.9-1.3\end{array}$ \\
\hline$\leq 64 \mathrm{~h}$ & $140 / 349$ & $\begin{array}{l}1.0 \\
0.8-1.3\end{array}$ & $27 / 70$ & $\begin{array}{l}1.0 \\
0.6-1.7\end{array}$ & $0 / 0$ & - & $167 / 419$ & $\begin{array}{l}1.0 \\
0.8-1.3\end{array}$ \\
\hline$>64 \mathrm{~h}$ & $80 / 232$ & $\begin{array}{l}1.1 \\
0.8-1.6\end{array}$ & $40 / 107$ & $\begin{array}{l}1.4 \\
0.9-2.1\end{array}$ & $8 / 18$ & $\begin{array}{l}1.3 \\
0.5-3.2\end{array}$ & $128 / 357$ & $\begin{array}{l}1.2 \\
0.9-1.5\end{array}$ \\
\hline Cordless & $167 / 437$ & $\begin{array}{l}1.0 \\
0.8-1.3\end{array}$ & $104 / 219$ & $\begin{array}{l}1.3 \\
1.01-1.8\end{array}$ & $23 / 45$ & $\begin{array}{l}1.6 \\
0.9-2.8\end{array}$ & $294 / 701$ & $\begin{array}{l}1.1 \\
0.9-1.4\end{array}$ \\
\hline$\leq 195 \mathrm{~h}$ & $98 / 260$ & $\begin{array}{l}1.0 \\
0.7-1.3\end{array}$ & $33 / 74$ & $\begin{array}{l}1.3 \\
0.8-2.0\end{array}$ & $3 / 17$ & $\begin{array}{l}0.9 \\
0.2-3.2\end{array}$ & $134 / 351$ & $\begin{array}{l}1.0 \\
0.8-1.3\end{array}$ \\
\hline$>195 \mathrm{~h}$ & $69 / 177$ & $\begin{array}{l}1.0 \\
0.7-1.4\end{array}$ & $71 / 145$ & $\begin{array}{l}1.5 \\
1.1-2.1\end{array}$ & $20 / 28$ & $\begin{array}{l}2.2 \\
1.2-4.2\end{array}$ & $160 / 350$ & $\begin{array}{l}1.3 \\
0.99-1.6\end{array}$ \\
\hline
\end{tabular}


Table II. Continued.

$\frac{>1 \text { - to 5-year latency }}{\mathrm{Ca} / \mathrm{Co} \quad \mathrm{OR}, \mathrm{CI}} \frac{\text { 5- to 10-year latency }}{\mathrm{Ca} / \mathrm{Co} \quad \mathrm{OR}, \mathrm{CI}} \quad \frac{\text { >10-year latency }}{\mathrm{Ca} / \mathrm{Co} \quad \mathrm{OR}, \mathrm{CI}} \frac{\text { Total, >1-year latency }}{\mathrm{Ca} / \mathrm{Co} \quad \mathrm{OR}, \mathrm{CI}}$

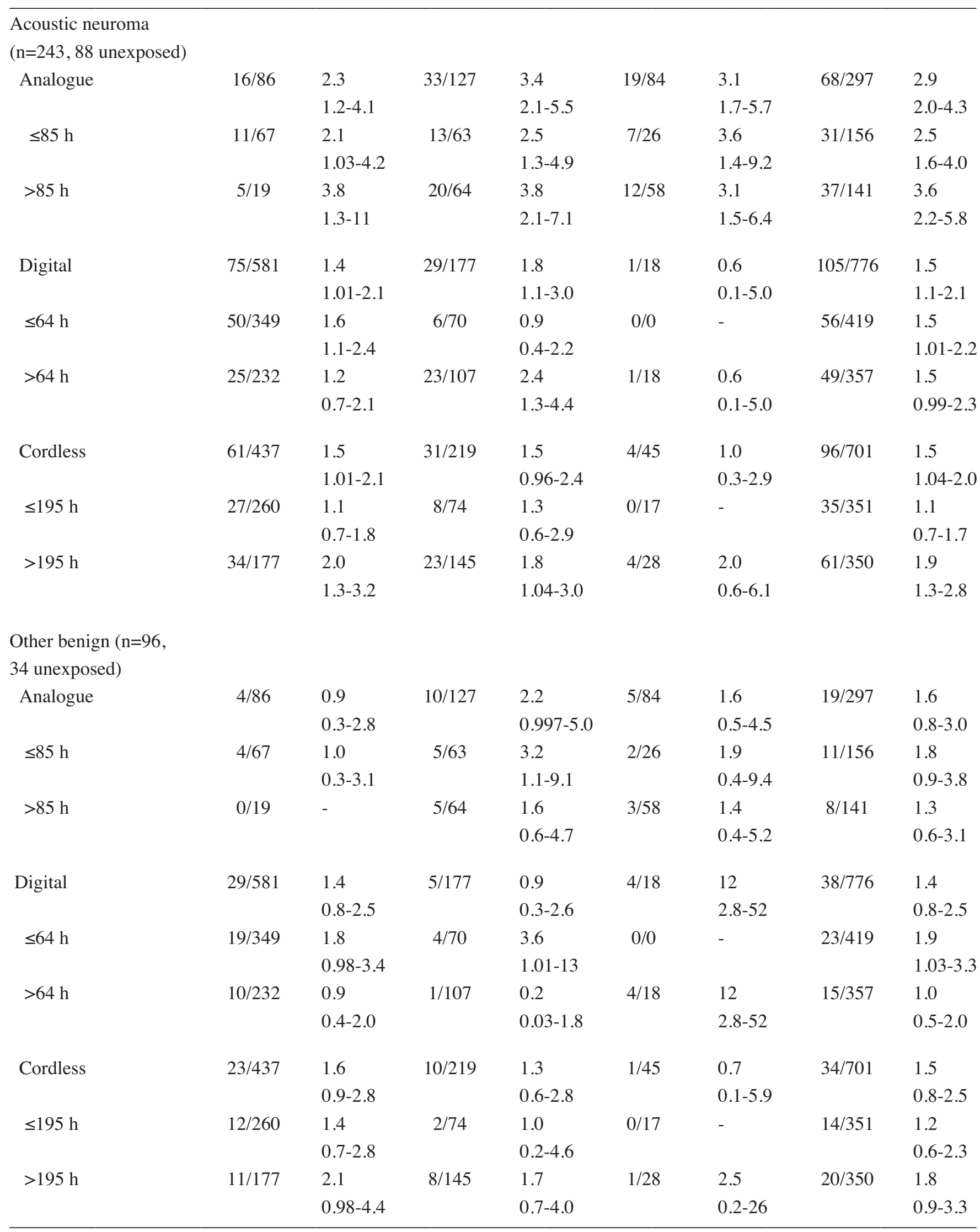

${ }^{a}$ Unconditional logistic regression analysis adjusted for age, sex, SEI and year of diagnosis, was used. In the dose-response calculations, the median number of cumulative use in hours among controls in the total material was used as a cut-off. 
Table III. Number of exposed cases (Ca) with benign brain tumours and controls (Co), odds ratio (OR) and 95\% confidence interval (CI) for use of cellular or cordless telephones for tumour localisations in relation to ear used during phone calls. ${ }^{a}$

\begin{tabular}{|c|c|c|c|c|}
\hline $\begin{array}{l}\text { Localisation/type } \\
\text { of telephone }\end{array}$ & $\begin{array}{c}\text { All } \\
\mathrm{Ca} / \mathrm{Co} \\
\mathrm{OR}(\mathrm{CI})\end{array}$ & $\begin{array}{c}\text { Ipsilateral } \\
\mathrm{Ca} / \mathrm{Co} \\
\mathrm{OR}(\mathrm{CI})\end{array}$ & $\begin{array}{c}\text { Contralateral } \\
\mathrm{Ca} / \mathrm{Co} \\
\mathrm{OR}(\mathrm{CI})\end{array}$ & $\begin{array}{c}\text { Ipsi-/contralateral } \\
\mathrm{Ca} / \mathrm{Co} \\
\mathrm{OR}(\mathrm{CI})\end{array}$ \\
\hline \multicolumn{5}{|l|}{ Benign } \\
\hline Analogue phone & $\begin{array}{l}199 / 297 \\
1.6 \\
1.3-2.0\end{array}$ & $\begin{array}{l}83 / 98 \\
1.9 \\
1.3-2.6\end{array}$ & $\begin{array}{l}59 / 100 \\
1.4 \\
0.99-2.1\end{array}$ & $\begin{array}{l}18 / 35 \\
1.3 \\
0.7-2.4\end{array}$ \\
\hline Digital phone & $\begin{array}{l}437 / 776 \\
1.2 \\
0.96-1.4\end{array}$ & $\begin{array}{l}172 / 240 \\
1.5 \\
1.1-1.9\end{array}$ & $\begin{array}{l}160 / 266 \\
1.2 \\
0.9-1.5\end{array}$ & $\begin{array}{l}45 / 84 \\
1.2 \\
0.8-1.9\end{array}$ \\
\hline Cordless phone & $\begin{array}{l}423 / 701 \\
1.2 \\
1.01-1.4\end{array}$ & $\begin{array}{l}167 / 232 \\
1.4 \\
1.1-1.8\end{array}$ & $\begin{array}{l}138 / 235 \\
1.1 \\
0.9-1.5\end{array}$ & $\begin{array}{l}42 / 77 \\
1.1 \\
0.7-1.7\end{array}$ \\
\hline \multicolumn{5}{|l|}{ Meningioma } \\
\hline Analogue phone & $\begin{array}{l}113 / 297 \\
1.3 \\
0.99-1.7\end{array}$ & $\begin{array}{l}42 / 98 \\
1.3 \\
0.9-2.0\end{array}$ & $\begin{array}{l}33 / 100 \\
1.2 \\
0.7-1.8\end{array}$ & $\begin{array}{l}10 / 35 \\
1.1 \\
0.5-2.3\end{array}$ \\
\hline Digital phone & $\begin{array}{l}295 / 776 \\
1.1 \\
0.9-1.3\end{array}$ & $\begin{array}{l}114 / 240 \\
1.4 \\
1.01-1.8\end{array}$ & $\begin{array}{l}112 / 266 \\
1.1 \\
0.8-1.5\end{array}$ & $\begin{array}{l}28 / 84 \\
1.1 \\
0.7-1.8\end{array}$ \\
\hline Cordless phone & $\begin{array}{l}294 / 701 \\
1.1 \\
0.9-1.4\end{array}$ & $\begin{array}{l}109 / 232 \\
1.3 \\
0.9-1.7\end{array}$ & $\begin{array}{l}101 / 235 \\
1.1 \\
0.8-1.5\end{array}$ & $\begin{array}{l}25 / 77 \\
0.9 \\
0.6-1.6\end{array}$ \\
\hline \multicolumn{5}{|l|}{ Acoustic neuroma } \\
\hline Analogue phone & $\begin{array}{l}68 / 297 \\
2.9 \\
2.0-4.3\end{array}$ & $\begin{array}{l}35 / 98 \\
3.0 \\
1.9-5.0\end{array}$ & $\begin{array}{l}23 / 100 \\
2.4 \\
1.4-4.2\end{array}$ & $\begin{array}{l}8 / 35 \\
2.3 \\
1.001-5.5\end{array}$ \\
\hline Digital phone & $\begin{array}{l}105 / 776 \\
1.5 \\
1.1-2.1\end{array}$ & $\begin{array}{l}50 / 240 \\
1.7 \\
1.1-2.6\end{array}$ & $\begin{array}{l}38 / 266 \\
1.3 \\
0.8-2.0\end{array}$ & $\begin{array}{l}16 / 84 \\
1.7 \\
0.9-3.2\end{array}$ \\
\hline Cordless phone & $\begin{array}{l}96 / 701 \\
1.5 \\
1.04-2.0\end{array}$ & $\begin{array}{l}52 / 232 \\
1.7 \\
1.1-2.6\end{array}$ & $\begin{array}{l}28 / 235 \\
1.1 \\
0.7-1.7\end{array}$ & $\begin{array}{l}15 / 77 \\
1.8 \\
0.9-3.4\end{array}$ \\
\hline \multicolumn{5}{|l|}{ Other benign } \\
\hline Analogue phone & $\begin{array}{l}19 / 297 \\
1.6 \\
0.8-3.0\end{array}$ & $\begin{array}{l}6 / 98 \\
4.3 \\
1.3-14\end{array}$ & $\begin{array}{l}3 / 100 \\
1.4 \\
0.3-5.9\end{array}$ & $\begin{array}{l}0 / 35 \\
- \\
-\end{array}$ \\
\hline Digital phone & $\begin{array}{l}38 / 776 \\
1.4 \\
0.8-2.5\end{array}$ & $\begin{array}{l}8 / 240 \\
1.7 \\
0.6-5.1\end{array}$ & $\begin{array}{l}10 / 266 \\
2.2 \\
0.8-6.0\end{array}$ & $\begin{array}{l}1 / 84 \\
0.6 \\
0.1-5.5\end{array}$ \\
\hline Cordless phone & $\begin{array}{l}34 / 701 \\
1.5 \\
0.8-2.5\end{array}$ & $\begin{array}{l}6 / 232 \\
1.7 \\
0.5-5.1\end{array}$ & $\begin{array}{l}9 / 235 \\
2.0 \\
0.7-5.5\end{array}$ & $\begin{array}{l}2 / 77 \\
1.5 \\
0.3-8.0\end{array}$ \\
\hline
\end{tabular}

apsilateral, same side for tumour and phone; contralateral, opposite side for tumour and phone; and ipsi-/contralateral, both ears used equally. Unconditional logistic regression analysis adjusted for age, sex, SEI and year of diagnosis, was used. Note that tumour site was missing for some cases and the matched control was excluded as well as controls with a missing corresponding case. 
Table IV. Number of exposed cases (Ca) and controls (Co), odds ratio (OR) and $95 \%$ confidence interval (CI) for the use of cellular or cordless telephones. ${ }^{a}$

\begin{tabular}{|c|c|c|c|c|c|c|c|c|}
\hline & \multicolumn{2}{|c|}{$>1$ - to 5-year latency } & \multicolumn{2}{|c|}{$>5$ - to 10 -year latency } & \multicolumn{2}{|c|}{$>10$-year latency } & \multicolumn{2}{|c|}{ Total, $>1$-year latency } \\
\hline & $\mathrm{Ca} / \mathrm{Co}$ & $\mathrm{OR}, \mathrm{CI}$ & $\mathrm{Ca} / \mathrm{Co}$ & $\mathrm{OR}, \mathrm{CI}$ & $\mathrm{Ca} / \mathrm{Co}$ & $\mathrm{OR}, \mathrm{CI}$ & $\mathrm{Ca} / \mathrm{Co}$ & $\mathrm{OR}, \mathrm{CI}$ \\
\hline \multicolumn{9}{|l|}{ Benign } \\
\hline Analogue & $52 / 86$ & $\begin{array}{l}1.2 \\
0.9-1.8\end{array}$ & $90 / 127$ & $\begin{array}{l}1.5 \\
1.1-1.9\end{array}$ & $57 / 84$ & $\begin{array}{l}1.5 \\
1.04-2.1\end{array}$ & $199 / 297$ & $\begin{array}{l}1.5 \\
1.2-1.8\end{array}$ \\
\hline Digital & $323 / 581$ & $\begin{array}{l}1.0 \\
0.9-1.2\end{array}$ & $101 / 177$ & $\begin{array}{l}1.1 \\
0.8-1.5\end{array}$ & $13 / 18$ & $\begin{array}{l}1.4 \\
0.7-3.0\end{array}$ & $437 / 776$ & $\begin{array}{l}1.0 \\
0.9-1.2\end{array}$ \\
\hline Cordless & $250 / 437$ & $\begin{array}{l}1.0 \\
0.8-1.2\end{array}$ & $145 / 219$ & $\begin{array}{l}1.2 \\
0.97-1.5\end{array}$ & $28 / 45$ & $\begin{array}{l}1.1 \\
0.7-1.8\end{array}$ & $423 / 701$ & $\begin{array}{l}1.1 \\
0.9-1.3\end{array}$ \\
\hline \multicolumn{9}{|l|}{ Meningioma } \\
\hline Analogue & $32 / 86$ & $\begin{array}{l}1.2 \\
0.8-1.8\end{array}$ & $47 / 127$ & $\begin{array}{l}1.1 \\
0.8-1.6\end{array}$ & $34 / 84$ & $\begin{array}{l}1.4 \\
0.9-2.1\end{array}$ & $113 / 297$ & $\begin{array}{l}1.2 \\
0.96-1.6\end{array}$ \\
\hline Digital & $220 / 581$ & $\begin{array}{l}1.0 \\
0.8-1.2\end{array}$ & $67 / 177$ & $\begin{array}{l}1.1 \\
0.8-1.5\end{array}$ & $8 / 18$ & $\begin{array}{l}1.2 \\
0.5-2.9\end{array}$ & $295 / 776$ & $\begin{array}{l}1.0 \\
0.8-1.2\end{array}$ \\
\hline Cordless & $167 / 437$ & $\begin{array}{l}0.9 \\
0.7-1.1\end{array}$ & $104 / 219$ & $\begin{array}{l}1.3 \\
0.98-1.6\end{array}$ & $23 / 45$ & $\begin{array}{l}1.4 \\
0.8-2.4\end{array}$ & $294 / 701$ & $\begin{array}{l}1.1 \\
0.9-1.3\end{array}$ \\
\hline \multicolumn{9}{|c|}{ Acoustic neuroma } \\
\hline Analogue & $16 / 86$ & $\begin{array}{l}1.7 \\
0.96-3.0\end{array}$ & $33 / 127$ & $\begin{array}{l}2.5 \\
1.7-3.9\end{array}$ & $19 / 84$ & $\begin{array}{l}2.2 \\
1.3-3.8\end{array}$ & $68 / 297$ & $\begin{array}{l}2.5 \\
1.8-3.5\end{array}$ \\
\hline Digital & $75 / 581$ & $\begin{array}{l}1.1 \\
0.8-1.6\end{array}$ & $29 / 177$ & $\begin{array}{l}1.5 \\
0.9-2.3\end{array}$ & $1 / 18$ & $\begin{array}{l}0.5 \\
0.1-3.9\end{array}$ & $105 / 776$ & $\begin{array}{l}1.1 \\
0.8-1.6\end{array}$ \\
\hline Cordless & $61 / 437$ & $\begin{array}{l}1.2 \\
0.9-1.7\end{array}$ & $31 / 219$ & $\begin{array}{l}1.1 \\
0.7-1.7\end{array}$ & $4 / 45$ & $\begin{array}{l}0.7 \\
0.2-1.9\end{array}$ & $96 / 701$ & $\begin{array}{l}1.1 \\
0.9-1.5\end{array}$ \\
\hline \multicolumn{9}{|l|}{ Other benign } \\
\hline Analogue & $4 / 86$ & $\begin{array}{l}0.7 \\
0.2-2.0\end{array}$ & $10 / 127$ & $\begin{array}{l}1.6 \\
0.8-3.2\end{array}$ & $5 / 84$ & $\begin{array}{l}1.1 \\
0.4-2.8\end{array}$ & $19 / 297$ & $\begin{array}{l}1.2 \\
0.7-2.0\end{array}$ \\
\hline Digital & $29 / 581$ & $\begin{array}{l}1.1 \\
0.7-1.7\end{array}$ & $5 / 177$ & $\begin{array}{l}0.6 \\
0.2-1.5\end{array}$ & $4 / 18$ & $\begin{array}{l}9.6 \\
2.6-36\end{array}$ & $38 / 776$ & $\begin{array}{l}1.1 \\
0.7-1.7\end{array}$ \\
\hline Cordless & $23 / 437$ & $\begin{array}{l}1.3 \\
0.8-2.1\end{array}$ & $10 / 219$ & $\begin{array}{l}1.1 \\
0.5-2.1\end{array}$ & $1 / 45$ & $\begin{array}{l}0.4 \\
0.05-2.9\end{array}$ & $34 / 701$ & $\begin{array}{l}1.1 \\
0.7-1.8\end{array}$ \\
\hline
\end{tabular}

${ }^{a}$ Unconditional logistic regression multivariate analysis adjusted for age, sex, SEI and year of diagnosis, was used.

\section{Discussion}

The reporting of new cancer cases to the Swedish cancer registry is compulsory. Also certain benign diseases such as benign brain tumours are reported. As soon as the histopathological diagnosis is obtained, the respective pathological departments send a report to the local cancer registry in the five medical regions in Sweden. In addition, the treating physician makes a clinical report. Thus, a high reporting frequency is obtained with good coverage of all new cases and no selection bias as to reporting exists.

We recruited cases in a consecutive way from cancer registries in the included medical regions, and we have no indication of selection bias in this respect. Thus, it was possible to include cases soon after diagnosis. For inclusion, it was necessary to have histopathological verification of the diagnosis. If information was unclear or missing in the cancer registry, we obtained copies of records from the pathology and radiology departments. Since all diagnoses were based on histopathology, it was possible for us to analyse different types of benign brain tumours as well as tumour localisation in the brain.

According to Table I, in our pooled study, it is obvious that a fairly high number of lifetime cumulative use of cellular or cordless telephones is necessary in order to obtain a stable risk estimate. 
Table V. Number of exposed cases (Ca) with benign brain tumours and controls (Co), odds ratio (OR) and 95\% confidence interval (CI) for the use of cellular or cordless telephones for different combinations of phone use. ${ }^{\mathrm{a}}$

\begin{tabular}{|c|c|c|c|}
\hline & \multicolumn{3}{|c|}{$>1$-year latency } \\
\hline & $\mathrm{Ca} / \mathrm{Co}$ & OR & $\mathrm{CI}$ \\
\hline \multicolumn{4}{|l|}{ Benign } \\
\hline NMT only & $45 / 79$ & 1.3 & $0.9-1.9$ \\
\hline GSM only & $168 / 312$ & 1.1 & $0.9-1.4$ \\
\hline Cordless only & $152 / 272$ & 1.0 & $0.8-1.3$ \\
\hline $\mathrm{NMT}+\mathrm{GSM}$ & $111 / 173$ & 1.6 & $1.2-2.2$ \\
\hline $\mathrm{NMT}+$ cordless & $113 / 138$ & 2.1 & $1.5-2.8$ \\
\hline GSM + cordless & $228 / 384$ & 1.3 & $1.05-1.7$ \\
\hline $\mathrm{NMT}+\mathrm{GSM}+$ cordless & $70 / 93$ & 2.0 & $1.4-2.9$ \\
\hline Total, any combination & $677 / 1172$ & 1.1 & $0.98-1.3$ \\
\hline \multicolumn{4}{|l|}{ Meningioma } \\
\hline NMT only & $26 / 79$ & 1.0 & $0.6-1.7$ \\
\hline GSM only & $119 / 312$ & 1.0 & $0.8-1.3$ \\
\hline Cordless only & $114 / 272$ & 1.0 & $0.8-1.3$ \\
\hline $\mathrm{NMT}+\mathrm{GSM}$ & $61 / 173$ & 1.3 & $0.9-1.8$ \\
\hline $\mathrm{NMT}+$ cordless & $65 / 138$ & 1.7 & $1.2-2.5$ \\
\hline GSM + cordless & $154 / 384$ & 1.2 & $0.9-1.6$ \\
\hline $\mathrm{NMT}+\mathrm{GSM}+$ cordless & $39 / 93$ & 1.7 & $1.1-2.6$ \\
\hline Total, any combination & $461 / 1172$ & 1.0 & $0.9-1.3$ \\
\hline \multicolumn{4}{|l|}{ Acoustic neuroma } \\
\hline NMT only & $11 / 79$ & 2.0 & $0.97-4.0$ \\
\hline GSM only & $33 / 312$ & 1.2 & $0.8-1.9$ \\
\hline Cordless only & $25 / 272$ & 1.0 & $0.6-1.6$ \\
\hline $\mathrm{NMT}+\mathrm{GSM}$ & $43 / 173$ & 3.3 & $2.0-5.3$ \\
\hline $\mathrm{NMT}+$ cordless & $42 / 138$ & 3.9 & $2.4-6.3$ \\
\hline GSM + cordless & $57 / 384$ & 1.6 & $1.04-2.4$ \\
\hline $\mathrm{NMT}+\mathrm{GSM}+$ cordless & $28 / 93$ & 4.1 & $2.3-7.1$ \\
\hline Total, any combination & $155 / 1172$ & 1.5 & $1.1-2.0$ \\
\hline \multicolumn{4}{|l|}{ Other benign } \\
\hline NMT only & $8 / 79$ & 2.0 & $0.8-4.7$ \\
\hline GSM only & $16 / 312$ & 1.5 & $0.7-2.8$ \\
\hline Cordless only & $13 / 272$ & 1.8 & $0.9-3.6$ \\
\hline NMT + GSM & $8 / 173$ & 1.3 & $0.5-3.3$ \\
\hline NMT + cordless & $7 / 138$ & 1.1 & $0.5-2.8$ \\
\hline GSM + cordless & $18 / 384$ & 1.5 & $0.8-3.1$ \\
\hline $\mathrm{NMT}+\mathrm{GSM}+$ cordless & 4/93 & 1.2 & $0.4-3.8$ \\
\hline Total, any combination & $62 / 1172$ & 1.5 & $0.9-2.4$ \\
\hline
\end{tabular}

${ }^{a}$ Unconditional logistic regression analysis adjusted for age, sex, SEI and year of diagnosis, was used.

In the analyses, we adjusted for sex, since all controls were used and they were frequency matched to the cases. It should be noted that meningioma more commonly occurs
Table VI. Odds ratio (OR) and $95 \%$ confidence interval (CI) in different age groups' first use of cellular or cordless telephones. $^{\text {a }}$

\begin{tabular}{lccc}
\hline & \multicolumn{3}{c}{$>1$-year latency } \\
\cline { 2 - 4 } & $\mathrm{Ca} / \mathrm{Co}$ & $\mathrm{OR}$ & $\mathrm{CI}$ \\
\hline Analogue phone & & & \\
All ages & $199 / 297$ & 1.6 & $1.3-2.0$ \\
$<20$ & $7 / 6$ & 3.9 & $1.2-12$ \\
$-20-49$ & $137 / 214$ & 1.6 & $1.2-2.1$ \\
$-50-80$ & $55 / 77$ & 1.5 & $1.03-2.2$ \\
Digital phone & & & \\
All ages & $437 / 776$ & 1.2 & $0.96-1.4$ \\
$<20$ & $6 / 9$ & 1.7 & $0.6-5.3$ \\
$-20-49$ & $250 / 445$ & 1.3 & $1.02-1.6$ \\
$-50-80$ & $181 / 322$ & 1.1 & $0.9-1.4$ \\
Cordless phone & & & \\
All ages & & & \\
$<20$ & $423 / 701$ & 1.2 & $1.01-1.4$ \\
$-20-49$ & $4 / 16$ & 0.6 & $0.2-1.9$ \\
$-50-80$ & $255 / 416$ & 1.4 & $1.1-1.7$ \\
\hline
\end{tabular}

${ }^{a}$ Numbers of exposed cases $(\mathrm{Ca})$ and controls $(\mathrm{Co})$ are given. Unconditional logistic regression analysis adjusted for age, sex, SEI and year of diagnosis, was used.

among females (13), so sex might be a confounder since the use of both cellular and cordless phones differs among men and women. The use is also age dependent and, generally, more common among younger persons, so adjustment was made for age in the calculations of OR and $95 \%$ CI. Another factor to take into account is the year of diagnosis for the cases and corresponding year for the controls since this pooled analysis encompassed cases and controls recruited during 1997-2003 and the use of both cellular and cordless telephones increases over the years. Finally, we also adjusted for current or last reported SEI-code since social class has been reported to be a determinant for brain tumours.

Different types of bias may limit the interpretation of results in all case-control studies. We designed the study to minimise this problem. We selected controls from the population registry to avoid selection bias in this respect. Blinding of case/control status in all data collection and coding of exposure avoided observation bias. Misclassification of exposure may occur if cases recall exposure different to controls. The questionnaire contained many questions other than use of cellular and cordless telephones in order to avoid focusing on such use. Furthermore, such a bias would occur for cases regardless of tumor type. Since our results differed for various types of benign brain tumours, it is less likely that the findings could be explained by recall bias. Also, the same methods and questionnaire were used in a study of salivary gland tumours where no association with cellular or cordless phones was found (14). 
The main finding of our pooled analysis of benign brain tumours was an increased risk for acoustic neuroma. The highest risk was found for a latency period of $>15$ years for use of analogue cellular telephones. However, an increased risk was also found for shorter latency periods. This might be consistent with a tumour-promoting effect from microwaves. In support of this, a Danish study found significantly larger acoustic neuroma among cellular phone users than controls (15).

In the univariate analysis, we found also a significantly increased risk for using digital cellular or cordless telephones. Furthermore, the risk was highest for ipsilateral use of the different phones, which certainly is of biological relevance. For analogue phones, increased ORs were also found for contralateral as well as varying ipsi- and contralateral use. This may be explained by the fact that hearing loss is an early symptom of acoustic neuroma leading to shift of the ear for phone calls during the progress of the disease. Thus, in spite of our efforts to carefully assess the most used ear over time, some cases may have reported the most recently used. It should be noted that only one case had used a digital phone and four cases a cordless phone with $>10$-year latency period. In the multivariate analysis, only analogue phones yielded a significantly increased risk for acoustic neuroma. So the association with digital cellular phones and cordless phones is still an issue for study with cases of long-term use.

For meningioma, we found an increased risk for analogue phones in the group with a $>10$-year latency period. However, no dose-response effect was seen. No significantly increased risk was found for digital cellular telephones. The OR increased for cordless phones with latency period with a tendency for dose-response effect. We found also some effect of laterality with the highest risk for ipsilateral exposure for all phone types. In the multivariate analysis, no significantly increased risks prevailed for meningioma.

As has been discussed elsewhere (1-3,9), the main shortcoming of most published studies on the association between cellular telephones and brain tumours has been that the latency period is too short. Thus, both longer latency periods and a higher cumulative number of hours of use are necessary in order to obtain a more precise estimate of the risk. In our pooled study, 64 cases with benign brain tumor had used a cellular telephone (analogue and/or digital) for $>10$ years, and it should be noted that 28 cases had used a cordless phone for $>10$ years. Regarding different types of benign brain tumours, the results were based on lower numbers. Thus, risk estimates for longer use of cellular or cordless telephones must be interpreted with caution.

Recently, results were presented from the Interphone (WHO) study on mobile phone use and risk of acoustic neuroma (12). The study consisted of one study in Sweden, Denmark, Norway and Finland and two studies in the UK. The main outcome was a significantly increased risk of acoustic neuroma with $\mathrm{OR}=1.8,95 \% \mathrm{CI}=1.1-3.1$ for analogue phone users after use for 10 years or longer. As we have discussed elsewhere, there are limitations in the design, conduct and interpretation of the Interphone study $(16,17)$. For example, interviews and coding of data were not blinded to the case/ control status of the subjects, cases were interviewed at hospitals and controls mostly at homes, controls were obtained from general practioners' practise lists in the UK and there was not a histopathological verification of diagnosis for all cases. For the Swedish part, the number of cases was not in agreement with the Swedish cancer registry $(16,17)$. Thus, the results may have been influenced by selection, recall and observation bias. These pitfalls were avoided in our studies as far as possible, as discussed in more detail elsewhere (6).

The mechanism for a carcinogenic effect from RF fields has been discussed for several years. Some studies have shown biological effects in experimental studies whereas these findings have not been replicated in others (1-3). Of interest are findings of genotoxic effects in cell systems exposed to radio frequency electromagnetic fields (RF-EMF) in the recently presented REFLEX-study (18). They used SAR levels that varied between 0.3 and $2 \mathrm{~W} / \mathrm{kg}$ and found an increase in single- and double-strand DNA breaks and micronuclei frequency. Findings of chromosomal aberrations were observed in fibroblasts (19) and an intracellular increase of free radicals in HL-60 cells. It was concluded that RF-EMF might activate several groups of genes that play a role in cell division, cell proliferation and cell differentiation. These results indicate pathophysiological mechanisms that could be a basis for the development of chronic diseases, such as cancer, in humans. Also, a recently published study in mice on radiofrequency exposure and cellular calcium homeostasis is of interest regarding carcinogenesis (20). The results suggested that carcinogenesis might be induced earlier and with different pathological forms in exposed mice than in controls. Based on these results and our findings, it must be concluded that the current allowed SAR level of $2 \mathrm{~W} / \mathrm{kg}$ based on thermal effects from RF-EMF is not appropriate.

In conclusion, this pooled analysis showed an increased risk of benign brain tumours, especially acoustic neuroma. For other types of benign brain tumours, no convincing pattern of association was found. For cellular telephones, the OR was highest for subjects with a first phone use at $<20$ years of age. However, several of the calculations were based on low numbers. This research area needs further study with increased numbers of exposed cases and higher latency periods.

\section{Acknowledgements}

This study was supported by grants from Cancer- och Allergifonden, Cancerhjälpen, Nyckelfonden, Örebro Cancer Fund.

\section{References}

1. Hansson Mild K, Hardell L, Kundi M and Mattsson M-O: Mobile telephones and cancer: is there really no evidence of an association? (Review). Int J Mol Med 12: 67-72, 2003.

2. Kundi M: Mobile phone use and cancer. Occup Environ Med 61: 560-570, 2004.

3. Kundi M, Hansson Mild K, Hardell L and Mattsson M-O: Mobile telephones and cancer - a review of epidemiological evidence. J Toxicol Environ Health B 7: 351-384, 2004.

4. Hardell L, Näsman Å, Påhlson A, Hallquist A and Hansson Mild K: Use of cellular telephones and the risk for brain tumours: a case-control study. Int J Oncol 15: 113-116, 1999.

5. Hardell L, Hansson Mild K, Påhlson A and Hallquist A: Ionising radiation, cellular telephones and the risk for brain tumours. Eur J Cancer Prev 10: 523-529, 2001.

6. Hardell L, Hallquist A, Hansson Mild K, Carlberg M, Påhlson A and Lilja A: Cellular and cordless telephones and the risk for brain tumours. Eur J Cancer Prev 11: 377-386, 2002. 
7. Hardell L, Hansson Mild K and Carlberg M: Further aspects on cellular and cordless telephones and brain tumours. Int J Oncol 22: 399-407, 2003.

8. Hardell L, Hansson Mild K, Sandström M, Carlberg M, Hallquist A and Påhlson A: Vestibular schwannoma, tinnitus and cellular telephones. Neuroepidemiology 22: 124-129, 2003.

9. Hardell L, Carlberg M and Hansson Mild K: Case-control study on cellular and cordless telephones and the risk for acoustic neuroma or meningioma in patients diagnosed 2000-2003. Neuroepidemiology 25: 120-128, 2005.

10. Hardell L, Carlberg M and Hansson Mild K: Case-control study of the association between use of cellular and cordless telephones and malignant brain tumors diagnosed during 2000-2003. Environ Res 2005, doi:10.1016/j.envres.2005.04.006.

11. Lönn S, Ahlbom A, Hall P and Feychting M: Mobile phone use and the risk of acoustic neuroma. Epidemiology 15: 653-659, 2004.

12. Schoemaker MJ, Swerdlow AJ, Ahlbom A, et al: Mobile phone use and risk of acoustic neuroma: results of the Interphone casecontrol study in five North European countries. Br J Cancer 93: 842-848, 2005.

13. Whittle IR, Smith C, Navoo P and Collie D: Meningiomas. Lancet 363: 1535-1543, 2004.
14. Hardell L, Hallquist A, Hansson Mild K, et al: No association between the use of cellular or cordless telephones and salivary gland tumours. Occup Environ Med 61: 675-679, 2004.

15. Christensen HC, Schüz J, Kosteljanetz M, et al: Cellular telephone use and risk of acoustic neuroma. Am J Epidemiol 159: 277-283, 2004

16. Hardell L and Hansson Mild K: Re: Cellular telephone use and risk of acoustic neuroma. Am J Epidemiol 160: 923, 2004.

17. Hansson Mild K, Hardell L and Kundi M: Mobile phone use and risk of intracranial tumors. Bioelectromagnetics Newsletter 183: 5-7, 2005.

18. REFLEX Risk Evaluation of Potential Environmental Hazards From Low Frequency Electromagnetic Field Exposure Using Sensitive in vitro Methods. http://www.itis.ethz.ch/downloads/ REFLEX_Final\%20Report_171104.pdf

19. Diem E, Schwarz C, Adlkofer F, Jahn O and Rüdiger H: Nonthermal DNA breakage by mobile-phone radiation $(1800 \mathrm{MHz})$ in human fibroblasts and in transformed GFSH-R17 rat granulosa cells in vitro. Mutat Res 583: 178-183, 2005.

20. Anghileri LJ, Mayayo E, Domingo JL and Thouvenot P: Radiofrequency-induced carcinogenesis: cellular calcium homeostasis changes as a triggering factor. Int J Radiat Biol 81: 205-209, 2005. 